\title{
USING ABM TO EVALUATE THE IMPACT OF SOCIAL NETWORKS ON CONSTRUCTION LABOUR PRODUCTIVITY
}

\author{
Daoud Kiomjian ${ }^{1}$, F. Jordan Srour ${ }^{2}$, and Issam Srour ${ }^{3}$
}

\begin{abstract}
Labour productivity depends on a wide variety of factors, some of which pertain to characteristics of the construction crews themselves. Several of these factors such as language and demographics are described in the literature as soft or intangible and are of stochastic nature. As such, traditional deterministic modelling techniques are not sufficient to capture the full picture of the factors that come into play when considering construction labour productivity. Agent based modelling $(\mathrm{ABM})$, a simulation technique with growing popularity, presents a powerful candidate for modelling construction sites due to its properties and ability to consider social aspects. This paper demonstrates that ABM is an acceptable paradigm for studying the effect of both tangible and soft features on construction labour productivity.
\end{abstract}

Keywords: Labour Productivity, Agent Based Modelling, Social Networks, Simulation.

\section{INTRODUCTION}

Labour productivity is an important factor to consider while planning and managing construction projects. However, due to the significant level of uncertainty associated with construction labour productivity, incorporating it in planning represents a major managerial challenge. Overcoming this challenge often necessitates using subjective methods, such as expert opinion, in order to provide accurate estimates for task completion times. More structured approaches include using industry standards for estimating productivity similar to the RSMeans references. Yet even these estimates assume uniformity among workers of a given trade and are based on historical data. A plethora of research has been conducted to evaluate the effect of individual factors on labour productivity.

Two major aims of the productivity literature are to help practicing engineers obtain better estimates of productivity and provide engineers with methods to improve the productivity of their crews. For example, Jarkas (2011) studied the effect of buildability on construction labour productivity, whereas Hanna et al. (2005) evaluated the connection between over staffing and productivity. Liu et al. (2010), on the other hand, detailed the effects of workflow variations on labour productivity. The majority of these

1 PhD Student, American University of Beirut, Department of Civil and Environmental Engineering, P.O. Box 1107-2020, Beirut, Lebanon, e-mail: dak18@mail.aub.edu

2 Assistant Professor, Lebanese American University, Department of IT and Operations Management, P.O. Box 13-5053, Chouran, Beirut 1102 2801, Lebanon, PH +961-1786456 x1235, e-mail: Jordan.srour@lau.edu.lb

3 Associate Professor, American University of Beirut, Department of Civil and Environmental Engineering, P.O. Box 1107-2020, PH +961-1350000 x3491 Beirut, Lebanon, e-mail: is04@aub.edu.lb 
works study the effect of a single factor, yet real life projects suggest that labour productivity behaves following a stochastic process dependent on multiple factors many of which are 'soft' in nature like demographics and gender and are thus difficult to quantify. The impact of a stochastic process coupled with the influence of multiple qualitative factors suggest using a higher class of modelling techniques similar to neural networks(Dissanayake et al., 2005) and simulation (Watkins et al., 2009, Sacks et al., 2013) to study construction labour productivity.

This paper demonstrates that Agent Based Modelling (ABM) is a technique that can incorporate a variety of stochastic and 'soft' factors to model labour productivity in the construction industry. The model presented in this paper is that of a prototype for a more complex Agent Based Model. The major contribution of this work is that it demonstrates the relevance of $\mathrm{ABM}$ as a modelling technique for studying productivity in the construction industry and the interplay of the factors which affect productivity. The outcome of this research is to provide an objective computational technique that accurately estimates productivity while realistically modelling the multitude of conditions which exist on construction sites. This is a step forward to integrate the pulled planning philosophy into construction project management through making information regarding the productivity and behaviour of construction crews available to the managers beforehand.

\section{BACKGROUND}

$\mathrm{ABM}$ is a simulation technique that studies the relationship between the micro level interactions among the individual agents and the emergent patterns of system wide phenomena (Bulleit and Drewek, 2012). ABM has witnessed popularity across various disciplines such as social sciences, economics, political sciences and marketing (Bonabeau, 2002). This paradigm has also succeeded in attracting attention within construction industry research. This popularity is evident through the increased volume of studies and publications dedicated to $A B M$ applications in the realm of the construction industry. For example, Ahn and Lee (2014) used ABM to study the effect of social norms and culture on absenteeism rates in the construction industry. Rojas demonstrated the usefulness of $\mathrm{ABM}$ tools as an instructional tool to introduce construction management students to issues they would encounter in real life projects. Ren et al. (2003) developed a multi-agent system that simulates the negotiation of construction claims. Most similar to our domain of interest -- productivity --Watkins et al. (2009), used ABM to evaluate the impact of crowding on construction productivity.

What makes $A B M$ attractive is its suitability for modelling complex and dynamic systems (Ahn and Lee, 2014); this is in line with the characteristics of the construction industry where constant change and variability are the norm (Sacks et al., 2013). Another interesting feature of ABM lies in its ability to model human interactions (Liang et al., 2016). The human factor plays a vital role in construction projects; however, the human element is difficult to model due to underlying stochasticity and the qualitative nature of such a factor (Liang et al., 2016). The individuals existing on site belong to different parties (engineer, contractor, $\mathrm{CM}$ ) and could have different interests (Liang et al., 2016). ABM offers itself as a modelling technique to properly simulate these interactions and understand their emergent effects at the project level.

$\mathrm{ABM}$ is also capable of representing complex systems without the need to make significant assumptions and simplifications(Bulleit and Drewek, 2012, Liang et al., 2016). Construction sites are complex environments dense with human interactions and 
significant task interdependence(Liang et al., 2016). Construction sites also include a multitude of disciplines, and therefore have a sizeable flow of large amounts of data originating from different disciplines. All these factors contribute considerably to increasing the complexity of site environments and dropping any one of these dimensions could have an impact on the accuracy of the modelling approach used. An agent based model allows for the inclusion of a wide array of these factors and could thus give researchers and practitioners a virtual reality insight into the factors and conditions existing on site. In turn, modifications can be made to policies and resource allocation decisions in order to optimize their utility, whether it is safety, productivity, cost or quality.

Although there seems to be a lack of consensus in the literature on the definition of an agent (Liang et al., 2016), multiple agent characteristics can still be identified. One agent characteristic with significant agreement in the literature is autonomy (Macal and North, 2005, Macal and North, 2014). When an agent is autonomous, it is able to take action on its own, and thus an agent has control over its behaviour (Jennings, 2000). The autonomy characteristic of agents is useful for modelling the decentralized aspect of construction sites. The decentralized aspect of the construction industry has been recognized in modern construction theory, and is considered a facet of the lean approach in construction management (Howell, 1999, Sacks et al., 2013). Although construction projects have a certain hierarchy where the project manager acts as the leader of the project, control over individual trades and activities is still done by the engineer or foreman in charge. In many cases, foremen have to make decisions on their own to solve certain problems, and the daily distribution of tasks to the labour crews is often done by the frontline personnel. Unexpected events or site conditions such as bad weather, could force project participants to deviate from the original planned schedule (Rojas and Mukherjee, 2006). Accordingly and in many cases, the centralized control or limitations which are typically exercised through schedules have to be modified, and unplanned events have to be managed on the spot by the site staff and not by the project manager.

In addition to being autonomous, agents are characterized by being heterogeneous, which means that the agent pool does not have to be uniform, and thus agents are identifiable from one another(Macal and North, 2005). This property matches the fragmented nature of the construction industry. Construction projects have multiple phases (design, construction, commissioning), that typically involve different actors with different interests. Even during the same phase multiple actors are involved. For example, electricians, plumbers, concrete masons, steel fixers and welders may be involved during the construction phase, and although many of these parties are dependent; they are entirely separate. Even among the same crew, workers with different cultural and educational backgrounds or experience levels exist. If the construction site is to be accurately represented within a computer environment, such factors should be considered.

Another important trait of agents is their adaptability which gives them the capability to change the rules governing their behaviour (Macal and North, 2009). Such a trait allows agents to learn and adapt to changes in their environment. As stated above, construction projects witness significant levels of variability, and agents modelling project participants should therefore be able to respond to changes in their environment. Moreover, construction project participants acquire knowledge throughout the course of the project which significantly affects the construction methods they use and, accordingly, their productivity. Agent based simulation of the construction industry should therefore include some sort of memory and should allow for the agents to adapt, 
learn and change throughout the course of the simulation in order to mimic the constantly changing environment at construction sites. Another important agent trait is the ability to communicate with one another(Macal and North, 2014). The capability of $\mathrm{ABM}$ to accommodate for non-numerical qualitative factors is considered one of its major advantages. A significant volume of information flows among the construction project participants, and this flow could assume both formal and non-formal modes. This significant communication coupled with the intensive human nature of the construction industry necessitates including the social aspect of the micro community existing on site, if accurate representations are to be sought.

In any construction site, communication paths could exist between any number of project participants; a formal one and an informal one. The formal connection pertains to the agent's location on the project's organizational chart. Such a connection affects the parties from which this agent receives directions and the ones to whom he might or might not issue orders.

The instruction or formal communication is modelled in our prototype ABM by the messaging capability embedded in the AnyLogic software package. An agent therefore sends a message to its peer, who will in turn check the contents of the message and act accordingly. The initial agent network connects members according to their crew affiliations, and therefore initially, the interactions among agents will be limited to exchanging instructions among one another. Yet, formal communication is not the only method for exchanging information on construction projects. Site personnel may also exchange information through informal communication.

The relevance of informal communication and information flow within the construction industry has been evaluated in multiple studies such as Malisiovas and Song (2014), Senescu et al. (2012), and Shohet and Frydman (2003). Communication problems are known to have a negative impact on productivity in culturally diverse construction sites, due to misunderstanding of instructions or information(Ling et al., 2012). Therefore, a worker might seek an informal explanation for his tasks from a fellow countryman, instead of going through the formal communication channels and asking his supervisor who could belong to a different culture or speak a different language. Informal communication could therefore be utilized by project managers in order to overcome the communication problems resulting from cultural diversity. Another important contribution offered by informal communication is that it decreases the average communication path length. If two workers S1 and S2 need to share a certain resource such as equipment, they could communicate directly without having to go through the foreman. Therefore, it is important to include informal communication in any model that attempts to study productivity in the construction industry. However, given the haphazard nature of informal communication, it does not occur in a structured and well organized path like its formal counterpart. As such, multiple paths could exist for informal communication. We believe that the original network topology that connects members according to their crews will be accompanied by a new emergent network which would come to existence during simulation. This network will be the one related to informal communication among agents. The emergent network, however, will be based on demographic markers and not on hierarchy. The model will demonstrate that workers tend to group and interact the most with individuals who share similar cultural characteristics, such as language. 


\section{ABM PROTOTYPE OF A CONSTRUCTION SITE}

In addition to ABM's capability in handling complexity through the agent characteristics noted in Section 2, ABM also enjoys a high-level of cognitive fit between the modelling environment and the real world. Figure 1 represents the analogy between a real life construction project and its equivalent ABM. As such, Figure 1 is divided into an upper and lower section one representing a real life construction site (below) and the other representing the construction site in silico(above). Figure 1 is further divided into a left and right section denoting, on the left, the site at time $t$ and on the right, the site at time $\mathrm{t}+1$. The three workers labelled $\mathrm{F} 1, \mathrm{~S} 1$, and $\mathrm{U} 1$ are represented as three circle shapes in the ABM layer. The ability to represent the three different types of workers within the $A B M$ layer highlights the heterogeneous aspect of $A B M$. The square shapes shown in the figure represent tasks; thus ABM is not just limited to human agents and can also be used to represent resources, tasks and equipment. The speech symbol denotes communication between the two workers F1 and S1 thereby capturing the social aspect of construction. The autonomy characteristic of $\mathrm{ABM}$ is depicted by giving the agent associated with worker U1 the choice to select between two tasks, through the flow chart embedded in the agent's logic. The adaptability characteristic of ABM is illustrated in the figure by providing a caption of the construction site and its model at two different times. The state of the task changed from being inactive to complete and such a change was reflected in changing the percent completion attribute of the task agent. The completion of this task also affected agent U1, who now has a smaller suite of tasks to select from. Such an update in the flow chart provides an additional illustration of the adaptability property of $\mathrm{ABM}$.

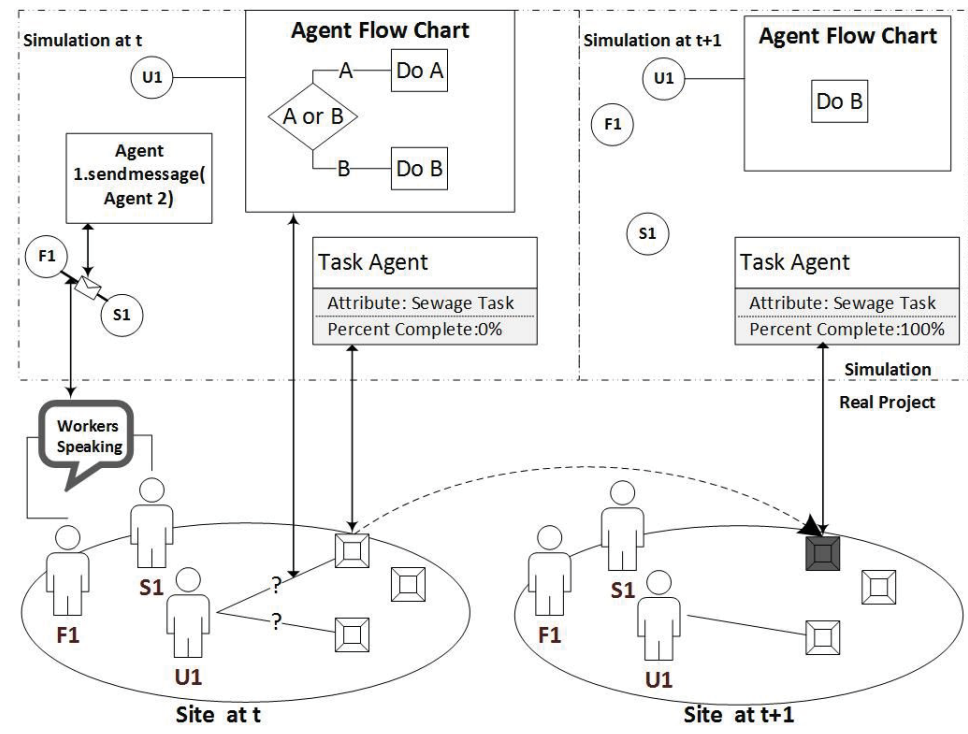

Figure 1: ABM simulation properties and real-life construction projects

\section{STATE CHARTS AND AGENT LOGIC}

The developed model includes two major agent categories: workers and tasks. Every worker agent has three parameters: a designation indicating whether it is a Foreman, Skilled worker, or Unskilled worker (helper); a productivity metric indicating the initial productivity of the agent in performing certain tasks; and a demographic marker. For illustration, the productivity of agents may be assigned stochastically according to a 
normal distribution. The demographic marker parameter is also stochastically assigned, but according to a uniform discrete distribution with four different values. As such, our model mimics a construction site with four different nationalities. A uniform distribution was used in order to ensure that the nationalities are distributed equally among the worker agents.

The worker agent can have one of three states: Idle which occurs when the agent is not executing any tasks; moving which is when the agent is moving towards his assigned task or when the agent is moving in search of a task; and working which is when the agent is working on his assigned tasks. The state chart for such an agent is shown in Figure 2: Worker agent statechart

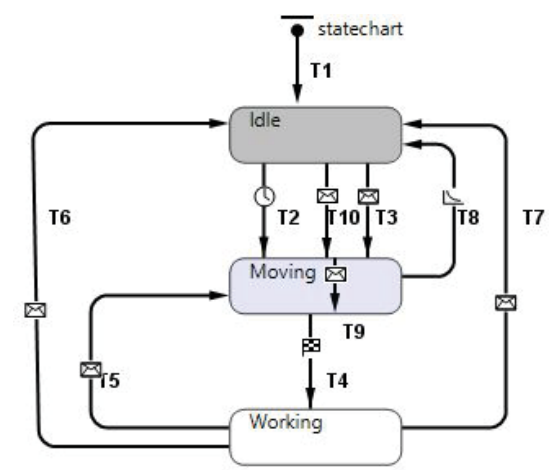

Figure 2: Worker agent statechart

Figure 2

The second type of transition is the communication triggered transitions, such as T3, T10, T5, T6, T7, and T9. The state changes dictated by these communication transitions are shown in Figure 3. This figure illustrates these transitions and represents their equivalent in a real life construction site. TransitionT3 and T10 occur when an agent receives, while being idle, an instruction/information from another agent that a certain task is currently available. The agent on the issuing side of the instruction might be a foreman, or a skilled agent. The agent would therefore start moving to get to this task and work on it. As the simulation runs for a longer period of time such a communication becomes more probable, if the two belong to the same demographic group. T5 on the other hand, occurs when an agent is working on a certain task and it receives an instruction from another agent with a foreman or skilled worker designation, to stop working the current task and move to another, thus changing the state of the worker agent from working to moving. T6 is similar to T5, however instead of instructing the working agent to move to another task, it is instructed to stay idle. T9 also shares some resemblance with the previous transitions, but in the case of $\mathrm{T} 9$, the worker agent receives the instruction from another agent with a superior designation, while moving to its target task. The worker agent alters its path in that it moves as per the received instruction. The final communication triggered transition is T7, this particular transition is different from the others since the agent on the sending end of the communication is the task agent, which needs to inform the worker agent that it is complete and thus no more work is necessary, which changes the state of the worker to idle. 


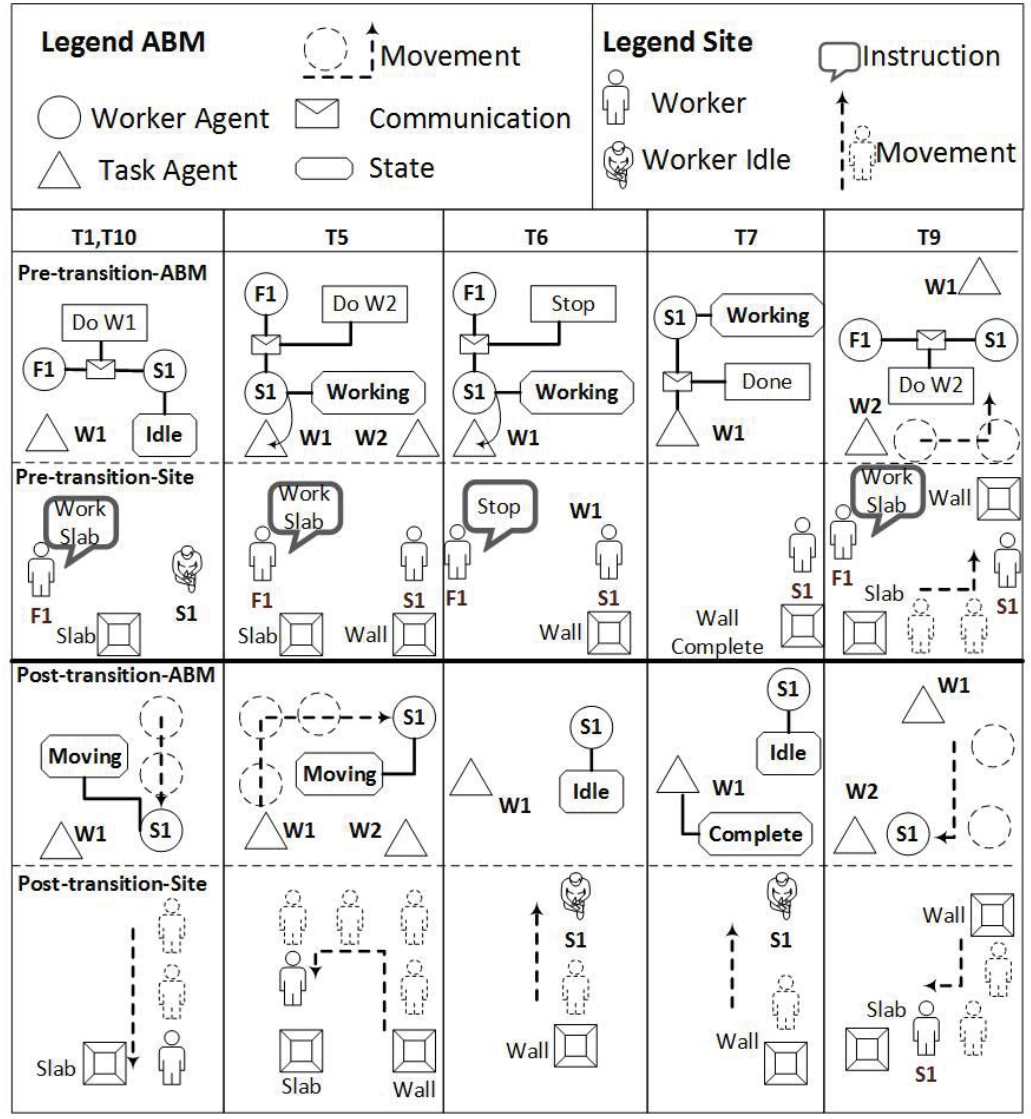

Figure 3: Communication triggered transitions

The task agent on the other hand has three states: Inactive, Active and Complete. When no worker is assigned to a certain task, the task is considered inactive; when a worker is working on a task, it is in the active state; and when no further work is needed for a certain task, it is considered complete. The task agent has one static parameter and one dynamic parameter. The static parameter is the size of the task which was selected to be 10 for all tasks; this was done to ease the prototyping process. However, future models will account for variability in task complexity and size. The dynamic parameter of the task is percentage complete and thus once this parameter reaches a value of 100 , the state of the relevant task agent changes from active to complete.

\section{CONCLUSIONS}

The model presented in this work is not a complete model for representing construction sites in the sense that it does consider resource availability on construction sites and different task sizes and complexities. Nevertheless, this model illustrates a possible method to simulate construction sites while considering a set of tangible and intangible factors. The model shows the different possible states for a worker on a construction site and demonstrates qualitatively the effect of team organization and worker communication on construction labour productivity. This paper explains different aspects of real construction sites such as varying labour demographics, autonomy, and agent communication, and how they can be represented in an agent based model. The results of this $\mathrm{ABM}$ will be validated by comparing the emergent properties of the model to observed phenomena on construction sites. Such results may provide insight regarding the effects of crew composition and information flow on productivity. The impact of 
having heterogeneous crews - in terms of culture and skill level - on site productivity could be investigated. Also, the positive or negative impacts of informal communication on the productivity of construction crews could be investigated.

Future modelling attempts could consider using a more developed environment for the agents such as GIS, and could incorporate resources such as vehicles and materials. Another factor to consider in future modelling attempts is the disciplines of the various tasks. Such a consideration would imply that not all workers can attempt to work on a certain task.

\section{ACKNOWLEDGMENTS}

We would like to express our gratitude to the University Research Board at the American University of Beirut for funding this research (Project Number: 23284). Any opinions, conclusions or findings expressed in this work are those of the authors and do not reflect the opinions of the University Research Board.

\section{REFERENCES}

Ahn, S. \& Lee, S. (2014). Methodology for creating empirically supported agent-based simulation with survey data for studying group behavior of construction workers. Journal of Construction Engineering and Management, 141, 04014065.

Bonabeau, E. (2002). Agent-based modeling: Methods and techniques for simulating human systems. Proceedings of the National Academy of Sciences, 99, 7280-7287.

Bulleit, W. M. \&Drewek, M. W. (2012) Agent-Based Modeling and Simulation for Hazard Management. Proc. Construction Research Congress (ASCE 2012). 2221-2230.

Dissanayake, M., Fayek, A. R., Russell, A. D. \& Pedrycz, W.A. (2005) hybrid neural network for predicting construction labour productivity. Proceedings of the International Conference on Computing in Civil Engineering, Cancun, Mex, 2005.1215.

Hanna, A. S., Chang, C., Lackney, J. A. \& Sullivan, K. T. (2005) Overmanning impact on construction labor productivity. Construction Research Congress. ASCE, 1-10.

Howell, G. A. (1999) What is lean construction. Proceedings IGLC.

Jarkas, A. M. (2011). Buildability factors influencing concreting labor productivity. Journal of Construction Engineering and Management, 138, 89-97.

Jennings, N. R. (2000). On agent-based software engineering. Artificial intelligence, 117, 277-296.

Liang, X., Shen, G. Q. \& Bu, S. (2016).Multiagent Systems in Construction: A Ten-Year Review. Journal of Computing in Civil Engineering, 04016016.

Ling, F. Y. Y., Dulaimi, M. F. \& Chua, M. (2012). Strategies for managing migrant construction workers from China, India, and the Philippines. Journal of Professional Issues in Engineering Education and Practice, 139, 19-26.

Liu, M., Ballard, G. \&Ibbs, W. (2010). Work flow variation and labor productivity: Case study. Journal of management in engineering, 27, 236-242.

Loosemore, M., Phua, F., Dunn, K. \&Ozguc, U. (2010). Operatives' experiences of cultural diversity on Australian construction sites. Construction Management and Economics, 28, 177-188.

Macal, C. \& North, M. Introductory tutorial: Agent-based modeling and simulation. Proceedings of the 2014 Winter Simulation Conference, 2014. IEEE Press, 6-20. 
Macal, C. M. \& North, M. J. Tutorial on agent-based modeling and simulation. Proceedings of the 37th conference on Winter simulation, 2005. Winter Simulation Conference, 2-15.

Macal, C. M. \& North, M. J. Agent-based modeling and simulation.Winter simulation conference, 2009. Winter simulation conference, 86-98.

Malisiovas, A. \& Song, X. Social Network Analysis (SNA) for Construction Projects' Team Communication Structure Optimization. Construction Research Congress 2014: Construction in a Global Network, 2014. 2032-2042.

Ren, Z., Anumba, C. \&Ugwu, O. 2003.Multiagent system for construction claims negotiation.Journal of Computing in Civil Engineering, 17, 180-188.

Rojas, E. M. \& Mukherjee, A. 2006.Multi-agent framework for general-purpose situational simulations in the construction management domain.Journal of Computing in Civil Engineering, 20, 165-176.

Sacks, R., Gurevich, U. \&Belaciano, B. 2013.Hybrid discrete event simulation and virtual reality experimental setup for construction management research.Journal of Computing in Civil Engineering, 29, 04014029.

Senescu, R. R., Aranda-Mena, G. \& Haymaker, J. R. 2012.Relationships between project complexity and communication. Journal of Management in Engineering, 29, 183-197.

Shohet, I. M. \&Frydman, S. 2003. Communication patterns in construction at construction manager level. Journal of construction engineering and management, 129, 570-577.

Watkins, M., Mukherjee, A., Onder, N. \&Mattila, K. 2009.Using agent-based modeling to study construction labor productivity as an emergent property of individual and crew interactions. Journal of construction engineering and management, 135, 657-667. 